\title{
Hypertrophic Pachymeningitis With Optic Neuropathy Heralding Systemic Vasculitis
}

\author{
Adrian Budhram, Manal Y. Gabril, Donald H. Lee, J. Alexander Fraser
}

Keywords: neuro-ophthalmology, vasculitis, meningitis, neuroinflammation, neuroimmunology, magnetic resonance imaging

A 47-year-old man presented with 1 year of right eye visual loss, headache, and mild bilateral nonpulsatile tinnitus. He had no other otologic, sinonasal, or systemic symptoms. On examination, visual acuity was finger counting in the right eye and 20/20 in the left eye. There was a right relative afferent pupillary defect and right optic disc pallor. Neurologic examination was otherwise normal. Gadolinium-enhanced magnetic resonance imaging scan of the head/orbits revealed T2-hyperintensity of the right optic nerve and predominantly right-sided dural thickening and enhancement, indicative of hypertrophic pachymeningitis (HP) (Figure 1). Inflammatory workup, including antineutrophil cytoplasmic antibody (ANCA) and immunoglobulin $\mathrm{G}$ ( $\mathrm{IgG}$ ) subtype testing, was ordered. Lumbar puncture was performed to send cultures for infection and cytology for malignancy.

The patient was otherwise well, so follow-up was arranged in 2 weeks to review results. Unfortunately, he presented emergently in the interim with a painful median neuropathy and acute renal failure. By then, his infectious and neoplastic workup had returned negative. Myeloperoxidase ANCA was positive at $187 \mathrm{RU} / \mathrm{ml}$ (cutoff $<20 \mathrm{RU} / \mathrm{ml}$ ) and erythrocyte sedimentation rate and $\mathrm{C}$-reactive protein were elevated, prompting initiation of high-dose steroids for presumed ANCA-associated vasculitis (AAV) with rapidly progressive glomerulonephritis. ${ }^{1}$ IgG subtyping returned soon thereafter: $\mathrm{IgG} 1$ and $\mathrm{IgG} 2$ were slightly elevated at $9.90 \mathrm{~g} / \mathrm{l}$ (cutoff $<9.30 \mathrm{~g} / \mathrm{l}$ ) and $7.33 \mathrm{~g} / \mathrm{l}$ (cutoff $<7.01 \mathrm{~g} / \mathrm{l}$ ), respectively; IgG3 was normal; and $\mathrm{IgG} 4$ was significantly elevated at $1.715 \mathrm{~g} / \mathrm{l}$ (cutoff $<0.865 \mathrm{~g} / \mathrm{l}$ ), warranting consideration of IgG4related disease (IgG4-RD). ${ }^{2}$ Renal biopsy was performed and revealed necrotizing crescentic glomerulonephritis, typical of AAV (Figure 2A). IgG4+ plasma cells were also present on immunohistochemical staining but numbered less than ten per high-powered field (Figure 2B), and as such the renal histopathology was felt to be more supportive of AAV than IgG4-RD. ${ }^{1,2}$

The patient rapidly developed mononeuritis multiplex, cardiac failure, pulmonary hemorrhage, and mesenteric ischemia, a clinical course highly compatible with systemic AAV. ${ }^{1}$ He underwent induction therapy with steroids, cyclophosphamide and plasma exchange before switching to rituximab because of gastrointestinal intolerance of cyclophosphamide, leading to disease stabilization and a slight visual improvement in the right eye.

\section{DISCUSSION}

$\mathrm{HP}$ is an inflammatory disorder that causes thickening of the dura mater and typically presents with headache, optic neuropathy, and other cranial neuropathies. ${ }^{3,4}$ It is most often diagnosed radiographically, and almost half of cases are idiopathic. ${ }^{4}$ ANCA positivity is the most common association with HP and suggests a forme fruste of $\mathrm{AAV}$, a necrotizing predominantly small-vessel vasculitis associated with myeloperoxidase-ANCA or proteinase 3 ANCA. ${ }^{1,3-5}$ The next most common cause of HP is IgG4-RD, an underrecognized fibro-inflammatory condition that likely accounts for a high proportion of cases previously considered idiopathic. $^{4,6,7}$ ANCA positivity with concomitant elevated IgG4 in HP is rare and may cause significant diagnostic uncertainty because elevated IgG4 has been described in diseases other than IgG4-RD, including $\mathrm{AAV}^{8}$; our case underscores the need to review all clinical, laboratory, and pathologic information in such patients to ensure accurate diagnosis. Remarkably, the latency from isolated HP with symptomatic optic nerve involvement to multisystem AAV in our patient was 1 year. Had our patient presented sooner, the earlier institution of immunosuppressive therapy may have mitigated his fulminant clinical course, highlighting the importance of recognizing this rare but distinct clinicoradiographic entity.

\section{STatement of Authorship}

$\mathrm{AB}$ and JAF examined the patient. MYG interpreted the histopathology slides. DHL interpreted the neuroimaging. All four authors drafted and revised the manuscript for intellectual content and created the figures together.

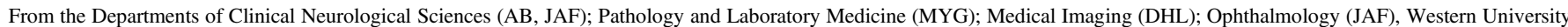
London, ON, Canada.

Received September 27, 2015. Revised February 21, 2016. Final Revisions Submitted March 11, 2016.

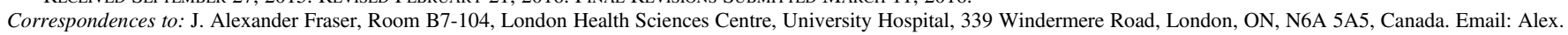
Fraser@lhsc.on.ca
} 

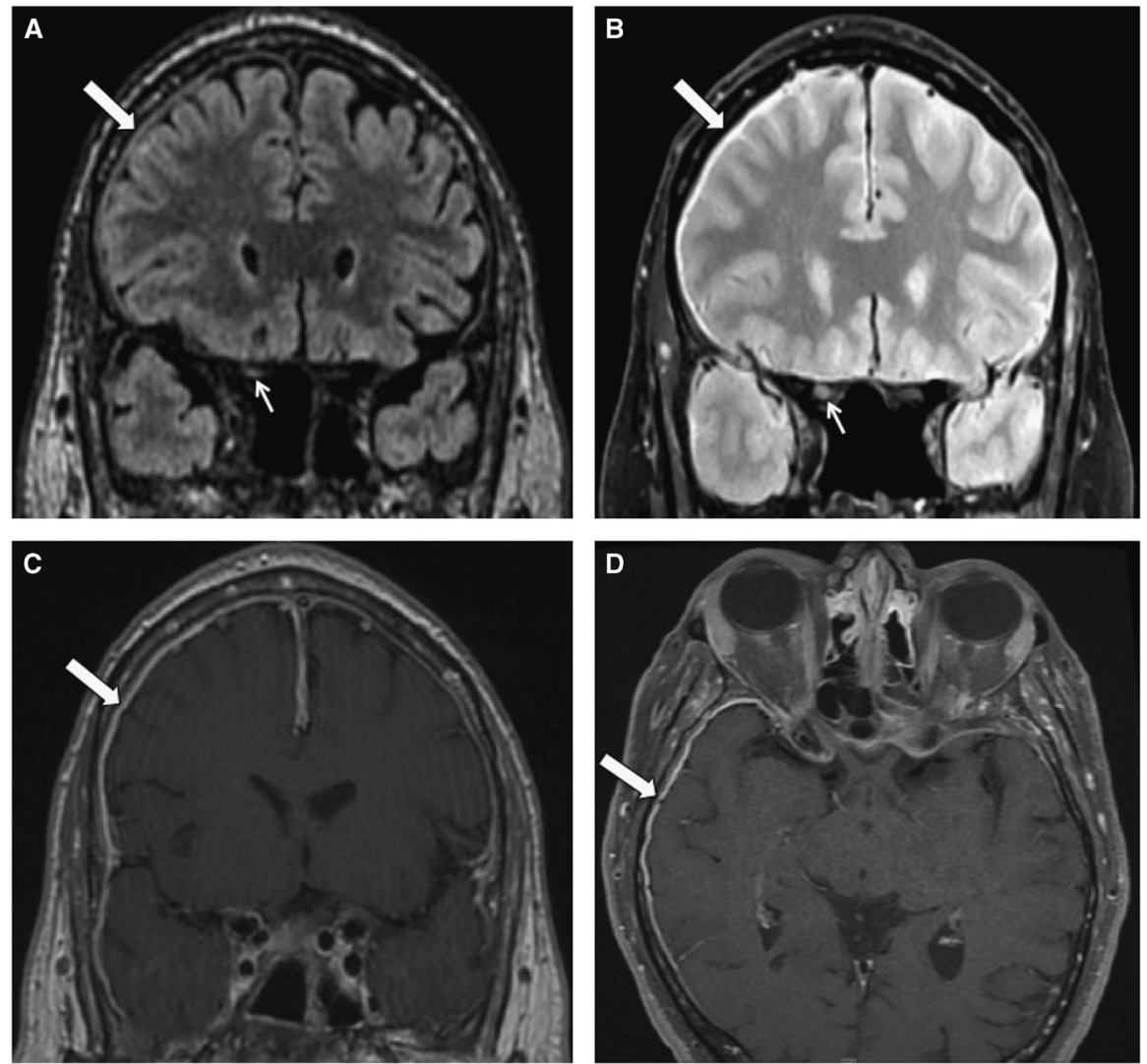

Figure 1: Magnetic resonance imaging (MRI) scan of the head (with gadolinium). MRI shows predominantly right-sided pachymeningeal thickening (long arrows) and right optic nerve hyperintensity (short arrows) on coronal fluid-attenuated inversion recovery $(A)$ and T2-weighted (B) images. Pachymeningeal enhancement (long arrows) is seen postgadolinium on coronal (C) and axial (D) T1-weighted images.
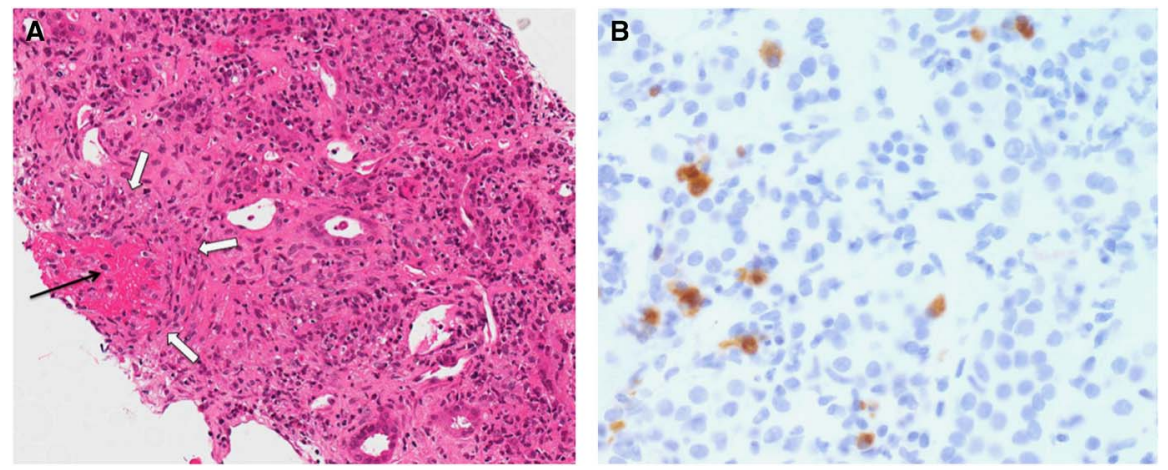

Figure 2: Histopathology of renal biopsy. Hematoxylin and eosin stain shows a glomerulus with segmental fibrinoid necrosis (black arrow) and a circumferential hypercellular crescent (white arrows) (A). Immunohistochemical staining for immunoglobulin G4-positive plasma cells reveals less than 10 per high power field (B).

\section{Disclosures}

None of the authors reports any disclosures.

\section{REFERENCES}

1. Jennette JC, Falk RJ, Bacon PA, et al. 2012 revised international Chapel Hill consensus conference nomenclature of vasculitides. Arthritis Rheum. 2013;65:1-11.
2. Umehara H, Okazaki K, Masaki Y, et al. Comprehensive diagnostic criteria for IgG4-related disease (IgG4-RD). Mod Rheumatol. 2012;22:21-30.

3. Yokoseki A, Saji E, Arkawa M, et al. Hypertrophic pachymeningitis: significance of myeloperoxidase anti-neutrophil cytoplasmic antibody. Brain. 2014;137:520-36.

4. Yonekawa T, Murai $\mathrm{H}$, Utsuki $\mathrm{S}$, et al. A nationwide survey of hypertrophic pachymeningitis in Japan. J Neurol Neurosurg Psychiatry. 2014;85:732-9. 
5. Li S, Tang H, Rong X, Huang X, Li Q. Pachymeningitis as a manifestation of ANCA-associated vasculitis: a case report and literature review. Int J Clin Exp Med. 2015;8:6352.

6. Popkirov S, Kowalski T, Schlegel U, Skodda S. ImmunoglobulinG4-related hypertrophic pachymeningitis with antineutrophil cytoplasmatic antibodies effectively treated with rituximab. J Clin Neurosci. 2015;6:1038-40.
7. Lu LX, Della-Torre E, Stone JH, Clark SW. IgG4-related hypertrophic pachymeningitis: clinical features, diagnostic criteria, and treatment. JAMA Neurol. 2014;6:785-93.

8. Ryu JH, Horie R, Sekiguchi H, Peikert T, Yi ES. Spectrum of disorders associated with elevated serum IgG4 levels encountered in clinical practice. Int J Rheumatol. 2012; 2012:232960. http://dx.doi.org/10.1155/2012/232960 